\title{
Dissecting catalysis
}

The catalytic mechanism of iterative polyketide synthases (IPKSs) is now more clearly understood thanks to the work of Townsend, Kelleher, Crawford and colleagues. Reporting in Science, they describe how - by using product analysis and mass spectrometry to characterize the intermediates that were bound to the fungal multidomain IPKS PksA following its dissection and reconstitution from selected sets of domains - they have been able to assign specific steps in the catalytic process to individual domains.

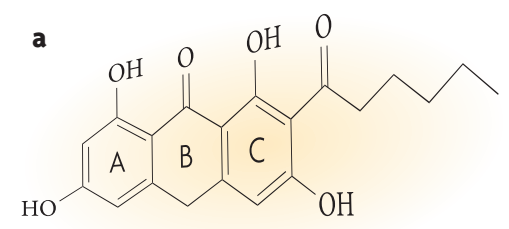

b

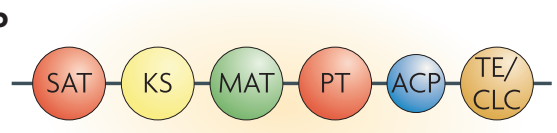

A catalytic product and the domain structure of PksA. a | Norsolorinic acid anthrone (NorA) is synthesized by PksA. The three rings in NorA are labelled A, B and C. $\mathbf{b}$ |The domain structure of $\mathrm{PksA}$ (see main text for acronym definitions).
Polyketides are a diverse range of natural compounds with varied biological activities and pharmacological properties. To improve our understanding of polyketide synthesis by IPKSs, the authors focused on Aspergillus parasiticus PksA, which initiates the biosynthesis of the environmental carcinogen aflatoxin $B_{1}$. Aflatoxin $B_{1}$ is a highly rearranged oxidation product of the much simpler molecule norsolorinic acid anthrone (NorA) (see the figure, part a), and all of the catalytic sites that are needed to make NorA are present in PksA. NorA is built from one unit of hexanoate and seven units of malonate, and the resulting polyketide chain undergoes cyclization reactions to form three adjoining rings with a hexanoate tail.

Crawford et al. were guided by the Udwary-Merski bioinformatics algorithm, which was previously used to identify the six-domain architecture of PksA (see the figure, part b). In their latest study, they used this information to dissect $\mathrm{PksA}$ into monodomains and various domain combinations. They then recombined the constructs in vitro and analysed the products formed. Their aim was to elucidate the domains that control polyketide chain length, the cyclization of the extended intermediate and product release.
They found that the ketosynthase (KS) domain controls the extension of a specific starter unit (hexanoate) that is attached to the acyl carrier protein (ACP) domain and elongates the polyketide chain to a fixed length. The product template (PT) domain subsequently drives the cyclization of the first two rings (see A and B in the figure, part a), but has no role in the cyclization of the third and final ring (see $\mathrm{C}$ in the figure, part a). This function belongs to the thioesterase/ Claisen cyclase (TE/CLC) domain, which also mediates product release.

As the starter-unit-ACP transacylase (SAT) domain is known to transfer hexanoate onto PksA to initiate polyketide synthesis and the malonyl-CoA:ACP transacylase (MAT) domain recruits malonate units, we now have a complete view of how the individual domains of PksA function. The mechanistic features determined here should apply to IPKS catalysis in general and the insights provided by this dissection approach "...enable a rational strategy for engineering these enzymes to synthesize alternative products."

Rachel Smallridge

ORIGINAL RESEARCH PAPER Crawford, J. M. et al. Deconstruction of iterative multidomain polyketide synthase function. Science $\mathbf{3 2 0}$, 243-246 (2008) 